\title{
Self-Assembled Monolayer Coatings on Nanostencils for the Reduction of Materials Adhesion**
}

\author{
By Marius Kölbel, R. Willem Tjerkstra, Gyuman Kim, Jürgen Brugger, Cees J. M. van Rijn, \\ Wietze Nijdam, Jurriaan Huskens, ${ }^{*}$ and David N. Reinhoudt*
}

Nanostencils (shadow masks with submicrometer apertures in a thin silicon nitride membrane) are promising tools for the facile one-step generation of nanopatterns of various materials by physical vapor deposition. Evaporation through a shadow mask is accompanied by gradual clogging of the apertures due to adhesion of evaporated material. In order to reduce this effect, nanostencils were coated with alkyl and perfluoroalkyl self-assembled monolayers (SAMs). The formation and properties of SAMs on planar silicon nitride substrates were studied by contact angle goniometry, X-ray photoelectron spectroscopy (XPS), and atomic force microscopy (AFM). The SAMs are stable under evaporation of gold at various angles. SAM-coated nanostencils showed considerably less adhesion of gold compared to bare $\mathrm{Si}_{x} \mathrm{~N}_{y}$ stencils.

\section{Introduction}

The spiraling progress information technology (IT) has seen over the last decades was essentially driven by ever faster hardware miniaturization. In the near future, however, the semiconductor industries will reach the resolution limits of optical lithography, their current central technology. These limitations on one hand, and the development of scanning probe techniques and nanotechnology in general on the other, have led to various novel patterning methods, e.g., electron beam lithography (EBL), focussed ion beam (FIB), X-ray lithography, soft lithography, and the nanostencil technique. ${ }^{[1]}$

A nanostencil consists of a thin silicon nitride membrane with aperture patterns obtained by optical lithography, EBL, or FIB, and is used as a shadow mask for the physical vapor deposition (PVD) of metals, semiconductors, insulators, or certain organic molecules onto various substrates under high vacuum (HV) or ultra-high vacuum (UHV) conditions. The fabrication of nanostencils with circular or line-shaped apertures having widths down to $200 \mathrm{~nm}$ is now state of the art, and

[*] Dr. J. Huskens, Prof. D. N. Reinhoudt, Dr. M. Kölbel Laboratory of Supramolecular Chemistry and Technology MESA+ Research Institute, University of Twente PO Box 217, NL-7500 AE Enschede (The Netherlands) E-mail: smct@ct.utwente.nl; d.n.reinhoudt@ct.utwente.nl Dr. R. W. Tjerkstra,

Transduction Technology Group

MESA+ Research Institute, University of Twente

PO Box 217, NL-7500 AE Enschede (The Netherlands)

Dr. G. Kim, Dr. J. Brugger ${ }^{[+]}$

NanoLink, MESA+ Research Institute, University of Twente PO Box 217, NL-7500 AE Enschede (The Netherlands)

Dr. C. J. M. van Rijn, Dr. W. Nijdam,

Aquamarijn Micro Filtration B.V.

Beatrixlaan 2, NL-7255 DB Hengelo Gld. (The Netherlands)

[+] Present address: Microsystems Laboratory, École Polytechnique Fédérale de Lausanne, CH-1015 Lausanne, Switzerland.

[**] We thank Mark A. Smithers for SEM images, Albert van den Berg for XPS measurements and Frans Segerink for FIB experiments. The work was supported by the ATOMS project of the EU. further reduction of the aperture size down to 80 and $50 \mathrm{~nm}$ has been demonstrated. ${ }^{[1,2]}$

The principle of the nanostencil technique is shown in Figure 1. Shadow-mask evaporation technology has several advantages. It is a resistless technique that allows generation of patterns in a single step. Integration of an $x, y$-stage for sub-

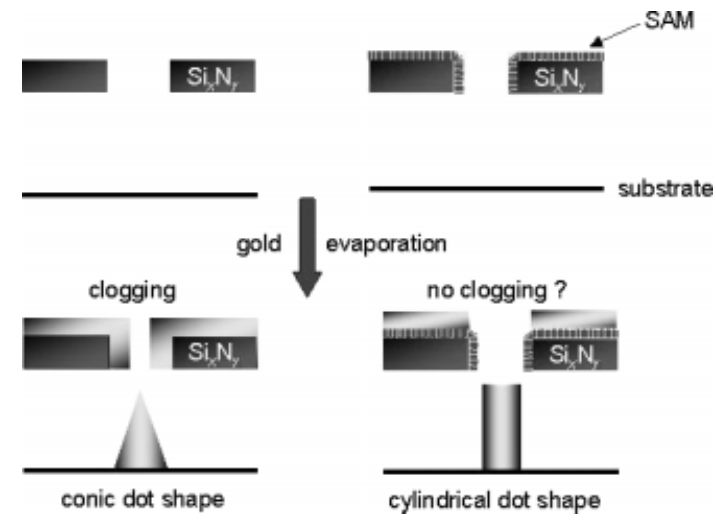

Fig. 1. Schematic representation of the nanostencil technique, the associated clogging problem, and the expected effect of SAM coatings.

strates allows generation of a nearly unlimited number of patterns using a single mask. Employing a multi-material source, a broad variety of materials can be deposited within one processing step. The nanostencil technique allows fast and parallel replication of patterns in a way comparable to optical lithography. Expensive and low-throughput techniques such as EBL or FIB are necessary only for the fabrication of the nanostencil. Last but not least, the nanostencil technique is environmentally friendly, since no etchants and processing liquids are needed, and less waste is produced. In principle, evaporated feature sizes are limited only by the aperture sizes of the stencil and thus by the limitations in their fabrication.

The schematic representation of the formation of circular metal dots given in Figure 1 shows a potential problem which may occur when working with a nanostencil: evaporated mate- 
rial is deposited not only on the substrate but also inside the apertures of the stencil, leading to their clogging. ${ }^{[1]}$ Frequent cleaning of the nanostencil using etchants or plasma treatment may solve this problem, but is undesirable since it requires a time-consuming interruption of the HV process. Preventing adhesion of material to the aperture walls and thus increase of the performance and throughput of the nanostencil technique is envisaged by coating the nanostencil with an anti-adhesion layer. We chose to coat the nanostencils with organic self-assembled monolayers (SAMs) and to investigate their influence on the adhesion properties of the stencil. The SAMs are formed by an irreversible chemical surface reaction of silanes with surface $\mathrm{OH}$ groups.

\section{SAMs on Silicon Nitride}

Literature on the surface chemistry of silicon nitride is mainly limited to the functionalization of silicon nitride atomic force microscopy (AFM) tips and their interactions with various substrates. Although chemical modification of planar $\mathrm{Si}_{x} \mathrm{~N}_{\mathrm{y}}$ surfaces with $\omega$-alkenyl-, $\mathrm{CH}_{3}-\mathrm{NH}_{2}$ - and $\mathrm{SO}_{3} \mathrm{H}$-terminated monolayers has been reported in two articles on tip-sample interactions, ${ }^{[3]}$ very few details on experimental conditions and monolayer stability that could be of potential value for our objective are given. Therefore we carried out some initial experiments on the formation of organic monolayers on $\mathrm{Si}_{x} \mathrm{~N}_{y}$ and studied their physical and chemical properties. ${ }^{[4]}$

Silicon nitride has a thin native $\mathrm{SiO}_{2}$ coating layer, and its surface can be activated by treatment with piranha solution $\left(\mathrm{H}_{2} \mathrm{SO}_{4} / 30 \% \mathrm{H}_{2} \mathrm{O}_{2}(\mathrm{aq}), 3: 1\right) .{ }^{[3]}$ Afterwards, the surface is composed mainly of silanol and silazane groups. ${ }^{[5]}$ Their ratio depends on various parameters, but at low $\mathrm{pH}$ and in the presence of water, mostly hydroxyl groups are found. ${ }^{[6]}$ These surface groups react with silane or germane molecules to give the desired monolayers.

Whereas the self-assembly of thiols on gold surfaces yields highly ordered monolayers and can usually be easily reproduced, formation of monolayers on silicon oxide (as bulk material or as native oxide layer on $\mathrm{Si}$ or $\mathrm{Si}_{x} \mathrm{~N}_{y}$ ) is less defined. ${ }^{[7]}$ The silane (or germane) compounds used are usually trifunctional and react not only with surface $\mathrm{OH}$ groups but also with neighboring molecules in the monolayer. This cross-linking increases the stability of the layers. However, since the degree of cross-linking is influenced by various factors (temperature, water content), reproducibility is poor. In particular, water plays an important role in the surface reaction: traces of water appear to be necessary in order to hydrolyze the $\mathrm{Si}-\mathrm{Cl}$ or $\mathrm{Si}-$ OR bonds of the silanes before the condensation reaction with surface $\mathrm{OH}$ groups can occur. ${ }^{[8]}$ However, silanols generated by this hydrolysis can also react with chloro- or alkoxy silanes in solution, giving polymer threads and globules which can contaminate the surface by physisorption or chemisorption (via non-polymerized functionalities). Thus, working under exclusion of moisture is indispensable in order to avoid contamination and to suppress the competing bulk polymerization reactions, but complete removal of water from solvents, equipment, and substrates can inhibit the desired surface reaction. The optimal conditions for the reproducible formation of high quality monolayers must therefore be established by a trial-and-error approach.

Reproducible and good-quality SAMs were obtained by immersion of freshly cleaned $\mathrm{Si}_{x} \mathrm{~N}_{y}$ substrates in 0.2 vol.- $\%$ solutions of the silanes or germanes (for compounds see Table 1) in dry $\mathrm{CH}_{2} \mathrm{Cl}_{2}$ at room temperature over a period of $16 \mathrm{~h}$, fol-

Table 1. Dynamic advancing and receding water contact angles for silane and germane $\mathrm{SAMs}$ on $\mathrm{Si}_{x} \mathrm{~N}_{y}$

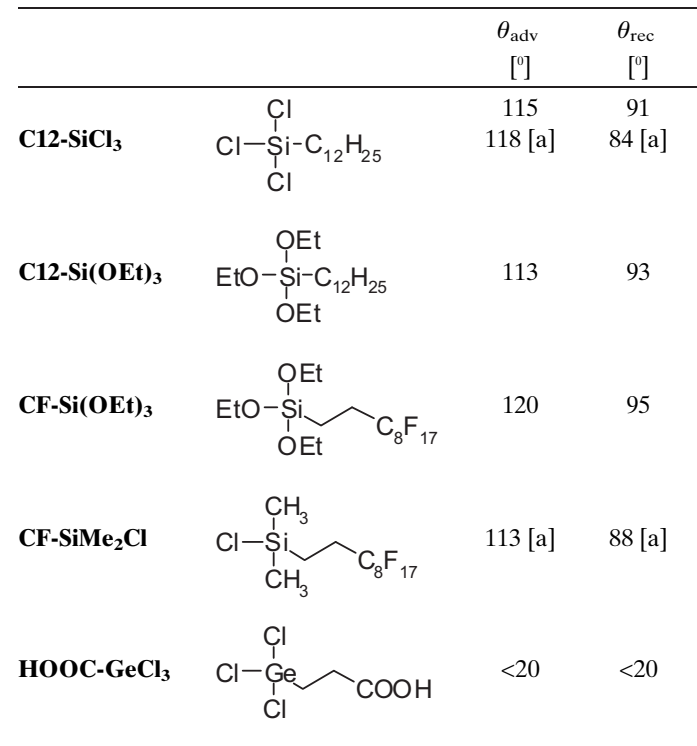

[a] Deposition from the gas phase.

lowed by extensive rinsing with organic solvents in order to remove physisorbed material. Compounds $\mathrm{C} 12-\mathrm{SiCl}_{3}$ and $\mathrm{CF}-\mathrm{Si}$ $\mathrm{Me}_{2} \mathrm{Cl}$ (because of its lower boiling point compared to $\mathrm{CF}-$ $\left.\mathrm{Si}(\mathrm{OEt})_{3}\right)$ were employed in an attempt to carry out vapor phase formation of a SAM. The resulting monolayers were studied by dynamic contact angle goniometry (Table 1), tapping-mode AFM, and X-ray photoelectron spectroscopy (XPS). Ellipsometry was attempted but appeared to be useless because of the basically trilayer ( $\mathrm{Si} / \mathrm{SiN} / \mathrm{SAM})$ structure of the coated substrates; small errors in the estimated SiN thickness caused errors in the determination of the SAM thickness larger than the SAM itself.

The water contact-angle data indicate hydrophobic surfaces with a hysteresis of $20-25^{\circ}$ for the alkyl and perfluoroalkyl SAMs deposited from solution. This is in accordance with values reported for silane SAMs on silicon oxide. ${ }^{[9]}$ Also the higher hydrophobicity of perfluorinated SAMs compared to hydrocarbon SAMs has been observed before. ${ }^{[10]}$ For $\mathrm{CF}-\mathrm{SiMe}_{2} \mathrm{Cl}$, a slightly less hydrophobic and less ordered SAM compared to $\mathrm{CF}-\mathrm{Si}(\mathrm{OEt})_{3}$ was observed. This is ascribed to a lower surface density of perfluoroalkyl chains due to the space required by the methyl groups. Nevertheless, the difference in SAM quality is small (also for $\mathrm{C} 12-\mathrm{SiCl}_{3}$ ) and gas phase deposition can thus be regarded as a valuable, alternative preparation method 
equivalent to solution deposition. The reduced demand of solvents and the exclusion of solution polymerization may make it preferable for some applications from a technical point of view. The low contact angle values measured for the SAM of the trichlorogermane $\mathrm{HOOC}-\mathrm{GeCl}_{3}$ indicate successful formation of a closely packed, highly hydrophilic SAM.

AFM showed smooth surfaces with very few thread-like or globular aggregates (height $\sim 5 \mathrm{~nm}$ ), most likely resulting from some solution polymerization. The roughness is comparable to that of bare silicon nitride surfaces (root-mean-square (RMS) roughness over $100 \mu \mathrm{m}^{2}$ for bare $\mathrm{Si}_{x} \mathrm{~N}_{y}$ is $0.66 \mathrm{~nm}$, for $\mathrm{C} 12-\mathrm{Si}(\mathrm{OEt})_{3}$-SAM $\left.0.83 \mathrm{~nm}\right)$.

Despite the XPS penetration depth of only $5 \mathrm{~nm}$, intense signals for $\mathrm{Si}$ and $\mathrm{N}$ were observed which can be ascribed to the $\mathrm{Si}_{x} \mathrm{~N}_{y}$ substrate. This confirms the absence of multilayers, as a monolayer has a thickness of $\sim 2 \mathrm{~nm}$. The atomic concentrations of $\mathrm{C}$ and/or $\mathrm{F}$ are in the expected range, although somewhat obscured by excess carbon, probably from atmospheric contamination. ${ }^{[11]}$

Since we chose the apolar compounds $\mathrm{C} 12-\mathrm{SiCl}_{3}, \mathrm{C} 12$ $\mathrm{Si}(\mathrm{OEt})_{3}$, and $\mathrm{CF}-\mathrm{Si}\left(\mathrm{OEt}_{3}\right)$ for coating the nanostencils, we also carried out experiments on their chemical and physical stability. We found (based on contact-angle measurements) that these monolayers can be stored at ambient conditions for months without changes in their properties. They are stable against repeated washings with organic solvents like $\mathrm{CH}_{2} \mathrm{Cl}_{2}$, ethanol, hexane, and toluene. No change in contact angles was detected after $24 \mathrm{~h}$ immersion in water and after storing the samples in an oven at $100^{\circ} \mathrm{C}$ for $2 \mathrm{~h}$. In basic cyanide gold etch solution the contact angle did not decrease for immersion times of up to $10 \mathrm{~min}$. Surprisingly, the SAMs are also relatively stable in piranha solution. The advancing water contact angle decreased $<5^{\circ}$ over $1 \mathrm{~h}$ for $\mathrm{CF}-\mathrm{Si}(\mathrm{OEt})_{3}$ and $\sim 10^{\circ}$ over $1 \mathrm{~h}$ for $\mathrm{C} 12-\mathrm{SiCl}_{3}$. The receding contact angles decreased by $10-15^{\circ}$ for both SAMs.

In order to check the stability of the SAMs under the conditions of metal evaporation we carried out an experiment with evaporation followed by etching (Fig. 2). In this experiment, SAMs of $\mathrm{C} 12-\mathrm{SiCl}_{3}$ and $\mathrm{CF}-\mathrm{Si}(\mathrm{OEt})_{3}$ were prepared on silicon nitride substrates. The advancing and receding water contact angles were found to be as listed in Table 1. One half of the substrate was masked with a silicon square. Then $40 \mathrm{~nm}$ gold was evaporated onto the unprotected region under an angle of $90^{\circ}$. The evaporation was carried out using a thermal evapora-
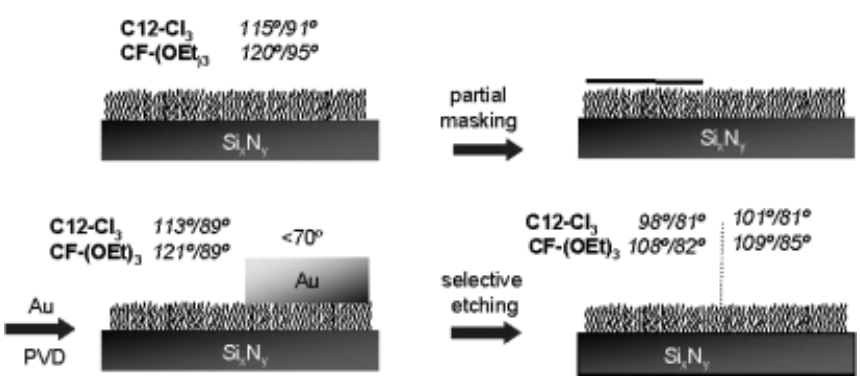

Fig. 2. Experiment on bombardment of SAMs with high-energy gold atoms advancing/receding dynamic water contact angles for SAMs of $\mathrm{C} 12-\mathrm{SiCl}_{3}$ and $\mathrm{CF}-\mathrm{Si}(\mathrm{OEt})_{3}$ after individual steps of the procedure. tor. The change in thickness of the gold layer formed was monitored by means of an internal quartz crystal microbalance (QCM). After this process, the contact angles of the uncoated region of the monolayer had not changed significantly, whereas the gold-coated area showed strongly reduced hydrophobicity. The gold was removed by selective etching $\left(\mathrm{K}_{3}\left[\mathrm{Fe}(\mathrm{CN})_{6}\right] /\right.$ $\left.\mathrm{K}_{4}\left[\mathrm{Fe}(\mathrm{CN})_{6}\right] / \mathrm{KOH} / \mathrm{Na}_{2} \mathrm{~S}_{2} \mathrm{O}_{3}, 15 \mathrm{~min}\right)$. Hereafter, the advancing contact angle was about $\sim 12^{\circ}$ lower on both areas. This change can probably be ascribed to the etch process. The absence of a difference between gold-covered and uncovered areas led us to conclude that bombardment with gold under $\mathrm{HV}$ conditions has no destructive impact on an alkylsilane or perfluoroalkylsilane monolayer. The slight damage to the SAM apparently caused by the etchant convinced us to use $\mathrm{I}_{2} / \mathrm{KI}$ etch solution in further experiments. SAMs on planar $\mathrm{Si}_{x} \mathrm{~N}_{y}$ substrates were found to be stable in this etchant without significant changes in contact angles for immersion times of at least $1 \mathrm{~h}$.

In order to better model evaporation inside the apertures, planar substrates with and without $\mathrm{CF}-\mathrm{Si}(\mathrm{OEt})_{3} \mathrm{SAM}$ were placed at angles of $45^{\circ}, 30^{\circ}$, and $0^{\circ}$ with the direction of the incoming gold. The last case represents lateral bombardment of the SAM, with the high-energy metal atoms attacking the molecules in the SAM, not at their end, but perpendicular to their chain, as is likely to be the case in the nanostencil apertures. After evaporation of $40 \mathrm{~nm}$ gold, the substrates placed at angles of $45^{\circ}$ and $30^{\circ}$ were covered with a layer of gold visible to the naked eye, for the SAM-coated substrates as well as for the untreated controls. For the substrates placed at $0^{\circ}$, however, no gold deposition was apparent. Water contact angle measurements at the SAM-coated sample showed no decrease in hydrophobicity $\left(\theta_{\mathrm{adv}}=120^{\circ}\right)$, but no distinct value for the receding contact angle could be measured, indicating that the order of the SAM had somewhat decreased. The latter observation can be ascribed to adhesion of small amounts of gold to the SAM-coated surface ( $3 \%$ according to XPS measurements). These results indicate that the SAMs are also stable under lateral bombardment.

\section{Experiments with Nanostencils}

The nanostencils used were made by silicon micromachining as shown in Figure 3. They consist of silicon nitride membranes with hexagonal arrays of holes having nominal diameters of $500 \mathrm{~nm}$, and spacings of $1 \mu \mathrm{m}$. The thickness of the membranes is $800 \mathrm{~nm}$. The holes have a tapered cross-section. Whereas on one side their diameters correspond to the nominal value, they are $605 \pm 35 \mathrm{~nm}$ on the other side (Fig. 4).

The SAM coating of the nanostencils was carried out in exactly the same manner as described above for planar substrates. In order to be able to clearly visualize differences in material deposition inside the stencil apertures, large amounts of gold (thickness $1 \mu \mathrm{m}$ ) were evaporated. The setup is shown in Figure 5. The nanostencils were placed on silicon substrates $(1 \mathrm{~cm} \times 1 \mathrm{~cm})$. The face with the smaller aperture diameters was positioned towards the gold source, the opposite face to- 

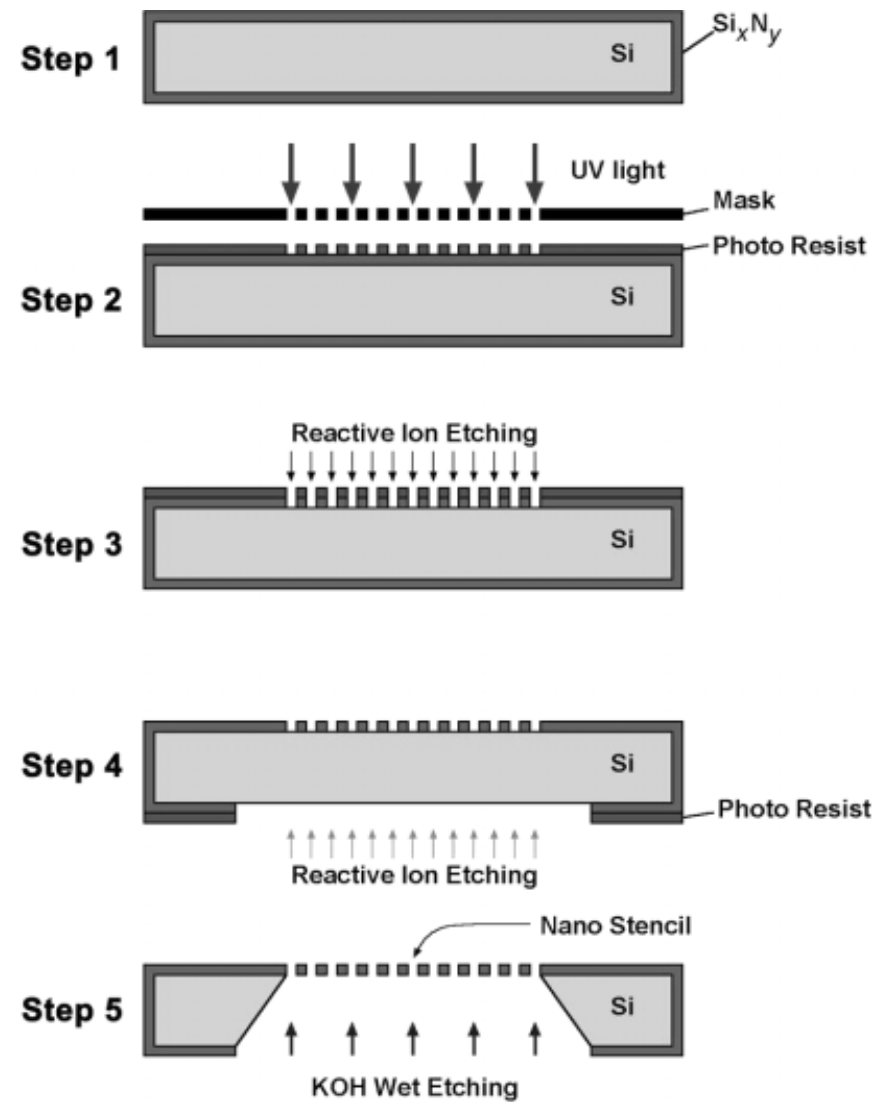

Fig. 3. Fabrication of the nanostencil.

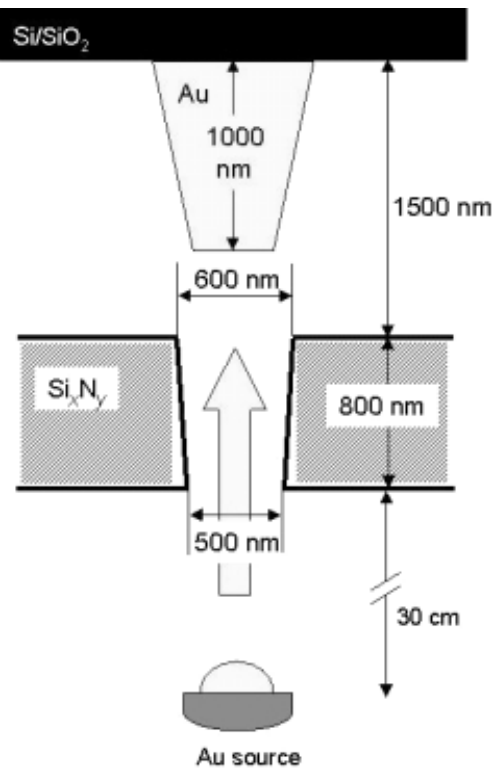

Fig. 4. Geometrical parameters of the nanostencils and the evaporation process.

wards the substrate. In order to prevent the micrometer dots from growing into the stencil apertures, a photoresist spacer ring of $1.5 \mu \mathrm{m}$ height was fabricated on the substrate. A flexible metal clamp was used to hold the samples in place on a sample holder, an aluminum plate with a diameter of 3 in. $(1 \mathrm{inch}=2.54 \mathrm{~cm})$ with windows for the samples. silicon substrate with a $1500 \mathrm{~nm}$ photoresist spacer ring

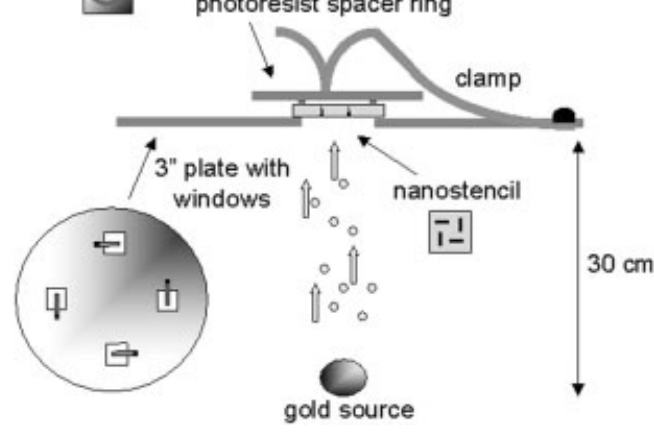

Fig. 5. Experimental setup for nanostencil evaporation experiments.

After the evaporations, the nanostencils were investigated by scanning electron microscopy (SEM). FIB etching was used to cut slits into the gold-covered membranes in order to image the cross-sections of the apertures by FIB itself (not shown) and by SEM under high tilt angles. ${ }^{[12]}$

Figure 6 shows SEM images of the nanostencil after evaporation of $1 \mu \mathrm{m}$ gold through $500 \mathrm{~nm}$ apertures. Clearly, the
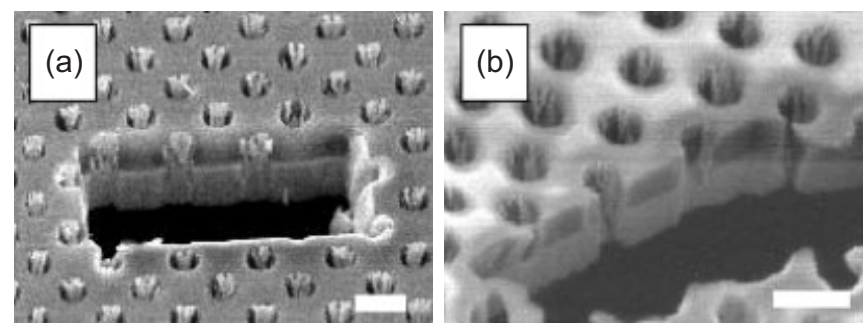

Fig. 6. SEM images of stencils after evaporation of $1 \mu \mathrm{m}$ Au. a) Uncoated $(15300 \times)$, b) $\mathrm{CF}-\mathrm{Si}(\mathrm{OEt})_{3}$ coated $(22000 \times)$; scale bars: $1 \mu \mathrm{m}$.

holes of the untreated nanostencil contain more gold than those of the $\mathrm{CF}-\mathrm{Si}(\mathrm{OEt})_{3}$ coated stencils. The pictures show that the gold forms long, leaf-shaped deposits in the holes of the former, indicating diffusion controlled growth, whereas only thin lines of gold are deposited inside the $\mathrm{CF}-\mathrm{Si}(\mathrm{OEt})_{3}$ coated apertures. The same difference with uncoated nanostencils was observed for the evaporation of $1 \mu \mathrm{m}$ gold through $500 \mathrm{~nm}$ apertures coated with $\mathrm{C} 12-\mathrm{Si}(\mathrm{OEt})_{3} \cdot{ }^{[13]}$

The improved sticking properties of the coated stencils prove that SAM formation has taken place inside the apertures as well as on the planar areas. The highly apolar monolayers strongly reduce the attractive interactions of the nanostencil surface with gold. Obviously, at the macroscopic outer surface, deposition does occur in any case because the gold hits the surface. In the nanoscopic pores, however, under the parallel flow of gold atoms, the reduced sticking appears to prevent the formation of gold clusters early in the process which can act as nucleation centers for the rapid growth of crystalline gold. This gold deposition inside the pores does, however, occur for the uncoated samples. Also, we observed that gold peels off easier from the SAM-coated stencils than from the uncoated ones. 

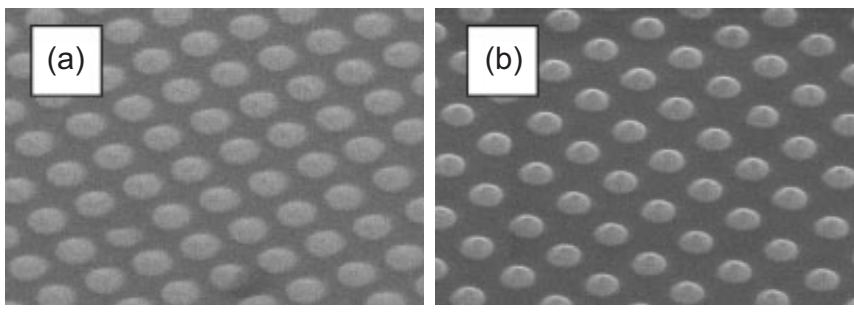

Fig. 7. SEM images of dot patterns formed following the evaporation of $150 \mathrm{~nm}$ Au through a) uncoated, and b) $\mathrm{CF}-\mathrm{Si}(\mathrm{OET})_{3}$-coated nanostencils with $350 \mathrm{~nm}$ pore diameters and a $1 \mu \mathrm{m}$ pitch.

Some evaporation results are shown in Figure 7, which shows SEM images of substrates after the evaporation of $150 \mathrm{~nm}$ of gold through such nanostencils, either uncoated (a) or coated with a $\mathrm{CF}-\mathrm{Si}(\mathrm{OEt})_{3}$ monolayer (b). The patterns for the uncoated stencil are clearly less well-defined and cover larger areas than patterns obtained with the coated stencil. More quantitative AFM analyses show almost a nominal height for patterns obtained with the coated stencil $(147 \mathrm{~nm})$, while a height of only $65 \mathrm{~nm}$ is observed for the patterns obtained with the uncoated stencil. Also the total dot volume appears to be a factor of two to three higher for coated stencils. A more quantitative description of the resulting patterns will be given elsewhere. ${ }^{[14]}$

Evaporation of material through the nanostencils inevitably leads to deposition on top of the nanostencil. This is particularly visible in Figure 6, which shows nanostencils after evaporation of $1 \mu \mathrm{m}$ of gold. This figure also shows that with such high loadings (or after repeated evaporations with a comparable total amount) the pores inside the built-up gold are smaller than the original stencil apertures. The consequences of this for the resulting patterns for such large evaporation amounts will be described elsewhere. ${ }^{[14]}$ Nevertheless, these results clearly show that cleaning is necessary after evaporating such amounts before re-use of the same nanostencils. The nanostencils could be cleaned easily by immersion in $\mathrm{I}_{2} / \mathrm{KI}$ gold etch solution $(0.2 \mathrm{~g}$ $\mathrm{I}_{2}, 3.15 \mathrm{~g} \mathrm{KI}$ in $50 \mathrm{~mL} \mathrm{H}_{2} \mathrm{O}$ ) for $\sim 10-20 \mathrm{~min}$ at room temperature. As mentioned earlier, the SAMs are stable under these conditions. No difference in etch rate between coated and uncoated nanostencils was observed.

\section{Conclusions}

We were able to show that silane SAMs on silicon nitride can be prepared and have properties comparable to those known from SAMs on silicon dioxide. The SAMs are stable against various physical and chemical influences and against vertical and lateral bombardment with gold vapor in HV. SAM coatings were expected to reduce adhesion of gold inside the apertures of silicon nitride nanostencil membranes. This effect is confirmed by SEM on nanostencils after gold evaporation: the images clearly show that less gold is deposited inside the pores of SAM-coated stencils compared to uncoated ones. These results show that SAM coatings can be used to improve performance and lifetime of nanostencils, thus increasing the throughput of this promising new nanopatterning technique.

\section{Experimental}

Chemicals and Instrumentation: Dodecyltrichlorosilane was purchased from Aldrich, dodecyltriethoxysilane and carboxyethyltrichlorogermane from ABCR, $1 \mathrm{H}, 1 \mathrm{H}, 2 \mathrm{H}, 2 \mathrm{H}$-perfluorodecyltriethoxysilane and $1 \mathrm{H}, 1 \mathrm{H}, 2 \mathrm{H}, 2 \mathrm{H}$-perfluorodecyldimethyltrichlorosilane from Lancaster. All compounds were used as obtained. Contact angles were determined using a Krüss Contact Angle Measurement System G10, and the data processed with the program Drop Shape Analysis 1.51. SEM images were taken with a Hitachi S800 FEG-SEM. A PHI Quantum 2000 scanning X-ray microprobe was employed for XPS investigations. FIB experiments were carried out by means of a FIB 200 (FEI Company).

Nanostencil Fabrication: The nanostencils used were made by silicon micromachining as shown in Figure 3. A thin layer (thickness $500 \mathrm{~nm}$ ) of low stress silicon nitride was deposited on a one side polished $\mathrm{Si}\langle 100\rangle$ wafer by Low Pressure Chemical Vapor Deposition $\left(\mathrm{SiH}_{2} \mathrm{Cl}_{2} / \mathrm{NH}_{3}=70: 18\right.$ at $850{ }^{\circ} \mathrm{C}, 250$ mbar) (step 1). A pattern with $500 \mathrm{~nm}$ pores in photo resist was made using mask photolithography (wafer stepper ASML PAS 5000/50) on the polished side of the silicon nitride (step 2). After development, this pattern was etched using reactive ion etching $\left(\mathrm{CHF}_{3} / \mathrm{O}_{2}=25: 5\right.$, power $=75 \mathrm{~W}$, pressure $\left.=10 \mathrm{mbar}, 6 \mathrm{~min}\right)$ creating pores in the silicon nitride (step 3 ). In step 4 , the process of lithography and reactive ion etching was repeated on the back side of the wafer, defining large rectangular openings in the silicon nitride. The silicon between the nanostencil layer and the back side was removed in an anisotropic etch step along the $\mathrm{Si}\langle 111\rangle$ planes with a $25 \% \mathrm{KOH}$ solution at $70^{\circ} \mathrm{C}$ until the membrane layer was reached (step 5). The nanostencils were diced at a rate of $2 \mathrm{~mm} \mathrm{~s}^{-1}$ (to avoid damage of the nanostencil) into square pieces of $5 \mathrm{~mm} \times 5 \mathrm{~mm}$ and cleaned in a $25 \% \mathrm{KOH}\left(70{ }^{\circ} \mathrm{C}\right)$ and a $5 \% \mathrm{H}_{2} \mathrm{SO}_{4}$ solution $\left(40^{\circ} \mathrm{C}\right)$ to remove all silicon particles that were released during dicing.

SAM Formation: All glassware was immersed in fresh piranha solution of $\sim 80^{\circ} \mathrm{C}$ for at least $1 \mathrm{~h}$, rinsed three times with milliQ water, blown dry with nitrogen, and stored in a glove box under $\mathrm{N}_{2}$ (not longer than $2 \mathrm{~h}$ ). The planar silicon nitride substrates $\left(150 \mathrm{~nm} \mathrm{Si}_{x} \mathrm{~N}_{y}\right.$ on $\left.\mathrm{Si}, 1 \mathrm{~cm} \times 1 \mathrm{~cm}\right)$ or nanostencils were treated with fresh piranha solution for $30 \mathrm{~min}$, rinsed three times with milliQ water, blown dry with nitrogen, and also stored in a glove box under $\mathrm{N}_{2}$ prior to use (not longer than $1 \mathrm{~h}$ ). Solutions were prepared immediately before use. $0.05 \mathrm{~mL}$ silane or germane were added to $25 \mathrm{~mL} \mathrm{CH}_{2} \mathrm{Cl}_{2}$ (freshly distilled from $\mathrm{CaCl}_{2}$ ) in a glass dish inside the glovebox. The substrates were immersed in the solution and the dish covered with a lid. After $16 \mathrm{~h}$ the substrates were transferred into a beaker containing $20 \mathrm{~mL}$ distilled $\mathrm{CH}_{2} \mathrm{Cl}_{2}$ and rinsed under shaking. The solvent was discarded and the rinsing repeated three times. After addition of another $20 \mathrm{~mL} \mathrm{CH} \mathrm{Cl}_{2} \mathrm{Cl}_{2}$, the substrates were taken out of the glovebox and rinsed again with two $20 \mathrm{~mL}$ portions of $\mathrm{CH}_{2} \mathrm{Cl}_{2}$, absolute $\mathrm{EtOH}$, and $\mathrm{CH}_{2} \mathrm{Cl}_{2}$. The substrates were then blown dry with $\mathrm{N}_{2}$. For gas phase deposition, the piranha-cleaned substrate was placed top-down above a dish containing $1 \mathrm{~mL}$ silane in a dessicator. The dessicator was evacuated to $\sim 10$ mbar, and after standing for $16 \mathrm{~h}$ at room temperature the substrate was removed and treated in the same manner as described for solution deposition.

Evaporations: The sample holder was placed in a Balzers BA 510 therma evaporator, $30 \mathrm{~cm}$ above and at an angle of $\sim 5^{\circ}$ to the evaporation source. The chamber was evacuated to $\sim 5 \times 10^{-6}$ mbar before the evaporation was started. The pressure during evaporation was $\sim 5 \times 10^{-5}$ mbar, the voltage $90 \mathrm{~V}$, the evaporation rate $\sim 24 \mathrm{~nm} \mathrm{~min}^{-1}$. The change in thickness of the gold layer formed was monitored by means of an internal quartz crystal microbalance (QCM).

Cleaning of the Stencils: The nanostencils were immersed in a solution of $0.2 \mathrm{~g}$ $\mathrm{I}_{2}$ and $3.15 \mathrm{~g} \mathrm{KI}$ in $50 \mathrm{~mL} \mathrm{H}_{2} \mathrm{O}$. The progress of the etching reaction was monitored in intervals of $5 \mathrm{~min}$ by optical microscopy after rinsing with milliQ water and drying the stencils in an $\mathrm{N}_{2}$ stream. 20 min was sufficient for complete removal of the gold

Received: May 6, 2002 Final version: September 6, 2002

[1] a) R. Lüthi, R. R. Schlittler, J. Brugger, P. Vettiger, M. E. Welland, J. K. Gimzewski, Appl. Phys. Lett. 1999, 75, 1314. b) J. Brugger, J. W. Berenschot, S. Kuiper, W. Nijdam, B. Otter, M. Elwenspoek, Microelectron. Eng. 2000, 53, 403. c) M. M. Deshmukh, D. C. Ralph, M. Thomas, J. Silcox, Appl. Phys. Lett. 1999, 75, 1631. d) R. R. Schlittler, J. W. Seo, J. K. Gimzewski, C. Durkan, M. S. M. Saifullah, M. E. Welland, Science 2001, 292 , 1136.

[2] C. J. M. van Rijn, G. J. Veldhuis, S. Kuiper, Nanotechnology 1998, 9, 343.

[3] a) T. Ito, M. Namba, P. Bühlmann, Y. Umezawa, Langmuir 1997, 13, 4323 b) V. V. Tsukruk, V. N. Bliznyuk, Langmuir 1998, 14, 446.

[4] M. Kölbel, J. Huskens, J. Brugger, D. N. Reinhoudt, Proc. Micro Nano Eng. Conf. 2001, Grenoble, September, 2001, p. 350.

[5] G. Busca, V. Lorenzelli, M. I. Braton, R. Marchand, J. Mol. Struct. 1986 143,525 . 
[6] a) L. Bergström, E. Bostedt, Colloids Surf. 1990, 49, 183. b) M. Okabe, S. Okazi, Nippon Kagaku Kaishi 1989, 10, 1802. c) Y. Li, Y. Gao, Y. Liang, F. Zheng, K. Xiao, Z. Hu, Wuli Huaxue Xuebao 1995, 11, 886.

[7] A. Ulman, Chem. Rev. 1996, 96, 1533.

[8] M. E. McGovern, K. M. R. Kallury, M. Thompson, Langmuir 1994, 10, 3607.

[9] A. Ulman, An Introduction to Ultrathin Organic Films, Academic Press, Boston, MA 1991.

[10] M.-W. Tsao, J. F. Rabolt, H. Schönherr, D. G. Castner, Langmuir 2000, 16 , 1734.

[11] The atomic concentration of $\mathrm{C}$ in $\mathrm{Si}_{x} \mathrm{~N}_{y}$ substrates when measured without pre-treatment was up to $40 \%$. XPS, carried out after 30 min cleaning in piranha solution, still gives $\sim 8 \% \mathrm{C}$.
[12] FIB cross-sections did not give reliable information since it was difficult to distinguish between gold deposited during the PVD process and gold re-deposited during the FIB etching. Our conclusions are based exclusively on SEM images of apertures to which no FIB had been applied.

[13] $\mathrm{C} 12-\mathrm{Si}(\mathrm{OEt})_{3}$ and $\mathrm{C} 12-\mathrm{SiCl}_{3}$ give $\mathrm{SAMs}$ with nearly identical properties The results obtained with $\mathrm{C} 12-\mathrm{SiCl}_{3}$ on stencils are, however, less reproducible. We assume this to be due to the higher reactivity and moisture sensitivity of the compound compared with $\mathrm{C} 12-\mathrm{Si}(\mathrm{OEt})_{3}$ and recommend always the use of alkoxy silanes.

[14] M. Kölbel, R. W. Tjerkstra, J. Brugger, C. J. M. van Rijn, W. Nijdam, J. Huskens, D. N. Reinhoudt, Nano Lett. 2002, 2, 1339. 